\title{
A Neural Network for Decision Making in Dynamic Environments
}

\author{
Abderrahim LABBI \\ LIFIA, Institut IMAG \\ 46, avenue Félix Viallet, \\ 38031 Grenoble cedex, France
}

\begin{abstract}
When designing a decision system (a pattern classifier, recognizer, or associator), we usually suppose that the information being processed by the system to compute a decision (output) remain unchanged (static) during the whole processing period called the System Autonomy Period (SAP). These systems are usually unable to process continuously dynamic information emerging from a changing environment since the internal states of such systems can not be forced to take into account sudden information changes in the environment. In this abstract is presented a competitive neural network which has the ability to process continuously dynamic information emerging from an unexpectedly changing environment. The network dynamics is described by a system of differential equations with external input $E$,
\end{abstract}

$$
\frac{d x_{i}}{d t}=-c_{i} \cdot x_{i}+\left(1-x_{i}\right) \cdot\left(\sum_{k=1}^{k=m} w_{i k} \cdot f_{k}\left(e_{k}\right)\right)-\left(1+x_{i}\right) \cdot\left(\sum_{j=1}^{n} d_{i j} \cdot g_{j}\left(x_{j}\right)\right) ; \quad i=1, \ldots, n
$$

To run a network represented by eq.(1), both $E$ and $X(0)$ must be specified. The input $E$ specifies the dynamics of the network, while $X(0)$ specifies the initial state of a trajectory obeying such dynamics. Each input vector $E$ induces a set of attractors $\Gamma(E)$ when the system (1) is supposed to be convergent. When $\Gamma(E)$ contains more than one attractor, the dynamics of a same $E$ may converge to different attractors depending on $X(0)$. Therefore, the network can not define a mapping from the environment to the attractor (decision) space. However, if for each $E, \Gamma(E)$ contains only one attractor (the system is sajed to be globally convergent), $X(0)$ does not matter, and the network can work as a pattern classifier or associator. If in addition, such attractor is stable, the system is saied to be globally asymptotically stable. By assuming that, $w_{i k} \geq 0, d_{i i}=0 \leq d_{i j}=d_{j i} \leq \alpha$, and $g_{j}(x)=\frac{1}{1+\exp (-x)}$, we have the following,

Theorem 1. The system (1) is convergent for any clamped input $E$.

Proof: By means of the variable change $z_{i}=1+x_{i}$, the system (1) can be written in the Cohen \& Grossberg form. $\square$

Theorem 2 . The system (1) is globally asymptotically stable for any clamped input vector $E$, provided, $c_{i} \geq(n-1) . \alpha ; i=1, \ldots, n$

Proof: available from the author. $\square$

Concerning learning, the network can be trained to classify a set of input data into classes by assigning a neuron in the decision layer to each class. The weights $w_{i k}$ can then be determined by a competitive learning rule. The network runs continuously having regard for the environment changes, and may recant and change its output without resetting its state variables when tending towards a first decision and new external changes occur.

A natural extension of the analysis of the dynamics (1) is to explore other conditions on the network parameters under which more complex behaviour occurs and generates more complex attractors such as limit cycles. Such study is being developed in the framework of bifurcation theory. 